\title{
On sufficient optimality conditions for multiobjective control problems
}

\author{
Valeriano Antunes de Oliveira ${ }^{1}$. Geraldo Nunes Silva ${ }^{1}$
}

Received: 1 February 2015 / Accepted: 13 August 2015 / Published online: 23 August 2015

C Springer Science+Business Media New York 2015

\begin{abstract}
This paper is devoted to presenting optimality conditions for the sufficiency of the maximum principle for multiobjective optimal control problems with nonsmooth data. Such conditions are the most general as possible in the sense that problems in which the set of necessary conditions from the maximum principle are also sufficient, necessarily obey them. A variation of such conditions is also presented, under which the set of optimal solutions of the multiobjective problem can be determined by resolving a related scalar weighting problem.
\end{abstract}

Keywords Optimal control · Optimality conditions · Generalized convexity · Multiobjective programming

Mathematics Subject Classification $\quad 49 \mathrm{~K} 15 \cdot 90 \mathrm{C} 26 \cdot 90 \mathrm{C} 29$

\section{Opening words}

The main purpose of this work is to provide sufficient optimality conditions for optimal control problems with multiple objective functionals in the nonsmooth setting. Necessary optimality conditions for optimal control problems are met in the well known Pontryagin Maximum Principle. This principle furnishes a set of first order conditions which lead to the characterization of optimal control processes candidates, the so called extremal (or stationary) control processes. The pioneer work is due to Pontryagin et al. [49]. See also Gamkrelidze [29] and Pontryagin et al. [50]. The nonsmooth maximum principle was developed in Clarke

$凶$ Valeriano Antunes de Oliveira antunes@ibilce.unesp.br

Geraldo Nunes Silva gsilva@ibilce.unesp.br

1 Instituto de Biociências, Letras e Ciências Exatas, Departamento de Matemática Aplicada, UNESP Universidade Estadual Paulista, Câmpus de São José do Rio Preto, Rua Cristóvão Colombo, N. 2265, São José do Rio Preto, SP CEP 15054-000, Brazil 
[16], Mordukhovich [44,45] and Vinter [58], for example. The impulsive case was treated, for example, in Silva and Vinter [54]. Another approach to necessary optimality conditions in the light of Gamkrelidze works [29,50] can be found in Arutyunov et al. [10,11] and Karamzin et al. [36]. Necessary optimality conditions for multiobjective optimal control problems were obtained, for instance, in Bellaassali and Jourani [12], Bonnel and Kaya [13], Kien et al. [39] and Zhu [60].

An extremal control process, as mentioned above, satisfies first order optimality conditions, the maximum principle conditions. Naturally, an extremal process may not be optimal. In order to distinguish a minimizing process among the extremals, we can resort, for example, to convexity conditions. The sufficient conditions provided in this work go into this direction. They are based on a kind of generalized convexity notion, called invexity.

It is well known that the Kuhn-Tucker necessary conditions turn to be also sufficient under convexity assumptions. Invex functions was designed in 1981 by Hanson [30] in order to get the sufficiency of the Kuhn-Tucker conditions for nonlinear programming problems. Later, Craven and Glover [19] showed that a differentiable real-valued function is invex if, and only if, every stationary point is a global minimizer. However, though, as demonstrated by Hanson, every stationary point is a global minimizer for invex mathematical programming problems, ${ }^{1}$ the converse is not true. That is, there are non invex problems with the property that every stationary point is a global minimizer. Therefore invex problems are not the most general class of problems which possesses such a property.

In 1985, Martin [41] redesigned Hanson's notion of invexity, while maintaining the sufficiency of the Kuhn-Tucker conditions. This generalized concept of invexity was termed KT-invexity. Moreover, Martin proved that if a mathematical programming problem is such that every stationary point is a global minimizer, then, necessarily, it satisfies this weakened invexity condition. Summarizing, Martin showed that every stationary point is a global minimizer if, and only if, the problem is KT-invex. Hanson [31] has also obtained some interesting results in this direction with the notion of type 1 invexity. See Zhao [59] and Reiland [51] for the nonsmooth case. Actually, Reiland [51] generalized the Hanson's paper [30] to include nonsmooth functions.

Martin's results were generalized for many other classes of optimization problems. See Arana-Jiménez et al. [9], Osuna-Gómez et al. [47,48] and Sach et al. [53] for multiobjective programming problems, Arana-Jiménez et al. [3,8] for variational problems, de Oliveira and Rojas-Medar [20,21,25] for continuous-time programming problems, and de Oliveira et al. $[24,26]$ for infinite programming problems. Non-regular optimization problems were studied in Hernández-Jiménez et al. [32-35].

The concept of KT-invexity was brought to the optimal control context in de Oliveira, Silva and Rojas-Medar [23] and Arana et al. [4,5]. The results in these papers were obtained assuming differentiability of the defining functions with respect to both state and control variables. Moreover, Lagrangian optimality conditions (Kuhn-Tucker type optimality conditions) were used instead of the maximum principle. In de Oliveira, Silva and Rojas-Medar [22] similar results were gotten for a multiobjective optimal control problem, still under smooth assumptions. In that article MP-pseudoinvexity was introduced and the maximum principle was employed. The scalar nonsmooth case was more recently tackled in de Oliveira and Silva [27]. Arana-Jiménez et al. [6,7] also treated multiobjective control problems with smooth Kuhn-Tucker type optimality conditions.

In this paper, MP-pseudoinvexity is generalized to include nonsmooth multiobjective optimal control problems. It is showed that every extremal process is optimal if, and only if,

${ }^{1}$ A problem is said to be invex if the defining functions are invex with the same invexity kernel. 
the problem is MP-pseudoinvex. We have then obtained similar results to those in [22] but for the nonsmooth setting. In addition, in [22], the concept of MP-invexity was introduced and some results on the so called scalarization method were established. This method consists in associating a mono-objective problem to the multiobjective one, where the objective function is a weighted sum of the coordinates of the vector-valued objective function. It was proved that when the multiobjective problem is MP-invex, an optimal solution of the multiobjective problem is an optimal solution of the weighted scalar problem, and conversely. Here, MPinvexity is likewise extended to the nonsmooth case. Analogous results are obtained.

Beyond type 1, KT and MP-invexity, many others types of invexities have appeared in the literature and applied to various classes of optimization problems. Concerning multiobjective optimization, we can cite Antczak [1,2], Mishra et al. [42,43] and Slimani [55,56].

Some interesting applications of invexity can be found in Dinuzzo et al. [28], Kenan and Lok [37,38], Nickisch and Seeger [46] and Syed et al. [57].

Another interesting class of non-convex problems where important properties of convex problems are preserved is given in Burai $[14,15]$. The author makes use of the concepts of bipolar (or second (Fenchel) conjugate) and connected (and quasi connected) functions.

A good reference on generalized convexity in multiobjective optimization problems is Luc [40]. Moreover, this book chapter brings an excellent bibliography list on the subject.

The paper is organized as follows. In the next section we give some background, formulate the multiobjective optimal control problem and state the maximum principle. Then, in Sect. 3, we define MP-pseudoinvexity and present some results on sufficient optimality conditions. The notion of MP-pseudoinvexity defined in Sect. 3.1 is appropriate for control problem for which the extremal processes do not degenerate, that is, for normal problems. Another definition of MP-pseudoinvexity is given in Sect. 3.2, suitable for general problems. Then we establish similar results to those for the normal case. In the last section we define MP-invexity and some results on the scalarization method for multiobjective problems are stated.

\section{Preliminaries}

We start by settling the notation which will be used throughout the text and retrieving some basic definitions. Then we formulate the nonsmooth multiobjective optimal control problem the paper is concerned with, lay down a set of assumptions that are necessary to establish our results, and present the maximum principle.

\subsection{Notation}

Given $v, w \in \mathbb{R}^{n}$, we denote the usual inner product between $v$ and $w$ as $v \cdot w$.

By $v \leq w$ we mean $v_{i} \leq w_{i}$ for all $i \in\{1,2, \ldots, n\}$; by $v<w$ we mean $v_{i}<w_{i}$ for all $i \in\{1,2, \ldots, n\}$; by $v \lessgtr w$ we mean $v_{i} \leq w_{i}$ for all $i \in\{1,2, \ldots, n\}$ and $v \neq w$.

Given a set $C \subset \mathbb{R}^{n}$, we denote the polar cone of $C$ by $C^{*}$, that is,

$$
C^{*}:=\left\{\zeta \in \mathbb{R}^{n}: \zeta \cdot v \leq 0 \quad \forall v \in C\right\}
$$

$\mathcal{L}$ denotes the Lebesgue subsets of a given interval $[S, T] ; \mathcal{B}^{n}$ denotes the Borel sets of $\mathbb{R}^{n}$; and $\mathcal{L} \times \mathcal{B}^{n}$ denotes the product $\sigma$-algebra.

Given a multifunction $\Gamma:[S, T] \rightarrow \mathbb{R}^{n}, \operatorname{Gr}(\Gamma)$ means the graph of $\Gamma$, i.e.,

$$
\operatorname{Gr}(\Gamma):=\left\{(t, \gamma) \in[S, T] \times \mathbb{R}^{n}: \gamma \in \Gamma(t)\right\}
$$


The set of all absolutely continuous functions $f:[S, T] \rightarrow \mathbb{R}^{n}$ is denoted by $W^{1,1}\left([S, T] ; \mathbb{R}^{n}\right)$.

\subsection{Basic definitions}

We now recall some definitions from nonsmooth analysis. For more details we refer the reader to one of the classical books on the subject, such as Clarke [16], Mordukhovich [44], and Vinter [58]. For a comprehensive treatment on nonsmooth analysis with applications to optimal control and variational analysis, we recommend Clarke et al. [17], Mordukhovich [45], and Rockafellar and Wets [52].

Given a closed set $C \subset \mathbb{R}^{n}$ and a point $x \in C$, the set of all directions $v \in \mathbb{R}^{n}$ such that there exists $M>0$ satisfying

$$
v \cdot(y-x) \leq M\|y-x\|^{2} \quad \forall y \in C,
$$

is said to be the proximal normal cone to $C$ at $x$, denoted by $N_{C}^{P}(x)$. The set of all directions $v \in \mathbb{R}^{n}$ such that there exist sequences $x_{i} \stackrel{C}{\rightarrow} x$ and $v_{i} \rightarrow v$ satisfying $v_{i} \in N_{C}^{P}\left(x_{i}\right)$ for all $i$, is said to be the limiting normal cone to $C$ at $x$, denoted by $N_{C}(x)$.

The set of all directions $w \in \mathbb{R}^{n}$ such that for any sequences $x_{i} \stackrel{C}{\rightarrow} x$ and $t_{i} \downarrow 0$ there exists a sequence $\left\{s_{i}\right\} \subset C$ satisfying $t_{i}^{-1}\left(s_{i}-x_{i}\right) \rightarrow w$, is said to be the Clarke tangent cone to $C$ at $x$, denoted by $\bar{T}_{C}(x)$.

The Clarke tangent cone and the limiting normal cone are related according to

$$
\bar{T}_{C}(x)=N_{C}(x)^{*} .
$$

Let $f: \mathbb{R}^{n} \rightarrow \mathbb{R} \cup\{+\infty\}$ be a lower semicontinuous function and $x \in \operatorname{dom} f$. By $\partial f(x)$ we mean the limiting Mordukhovich's subdifferential of $f$ at $x$ defined as the set

$$
\partial f(x)=\left\{\zeta:(\zeta,-1) \in N_{\text {epi } f}(x, f(x))\right\} .
$$

Let $L: \mathbb{R}^{n} \rightarrow \mathbb{R}^{m}$ be a Lipschitz function defined in a neighbourhood of $y \in \mathbb{R}^{n}$. By $D L(y)$ we mean the generalized Jacobian of $L$ at $y$ defined as the set of $m \times n$ matrices

$$
D L(y)=\operatorname{co}\left\{\vartheta: \exists y_{i} \rightarrow y \text { such that } \nabla L\left(y_{i}\right) \text { exists } \quad \forall i \text { and } \nabla L\left(y_{i}\right) \rightarrow \vartheta\right\} .
$$

Let $v \in \mathbb{R}^{n}$. The generalized Jacobian has the following interesting property:

$$
v^{\top} D L(y)=\operatorname{co} \partial(v \cdot L)(y) .
$$

\subsection{The multiobjective optimal control problem}

We deal with a multiobjective optimal control problem (MCP) posed as follows:

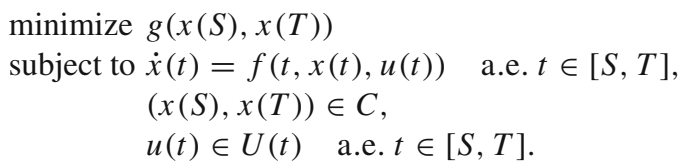

Here, $g=\left[g_{1} g_{2} \ldots g_{k}\right]^{\top}: \mathbb{R}^{n} \times \mathbb{R}^{n} \rightarrow \mathbb{R}^{k}, f:[S, T] \times \mathbb{R}^{n} \times \mathbb{R}^{m} \rightarrow \mathbb{R}^{n}, C$ is a closed subset of $\mathbb{R}^{n} \times \mathbb{R}^{n}, U$ is a multifunction mapping $[S, T]$ to nonempty subsets of $\mathbb{R}^{m}, S$ and $T$ are fixed.

We follow the classical nomenclature (see Vinter [58], for example), which is set below. 
A measurable function $u:[S, T] \rightarrow \mathbb{R}^{m}$ such that $u(t) \in U(t)$ a.e. $t \in[S, T]$ is said to be a control function.

A pair $(x, u)$ consisting of a control function $u$ and an $\operatorname{arc} x \in W^{1,1}\left([S, T] ; \mathbb{R}^{n}\right)$ obeying the differential equation above is called a process.

Given a process $(x, u)$, its first component is said to be a state trajectory.

We say that $(x, u)$ is a feasible process if $x$ is a trajectory corresponding to the control $u \in U$ which satisfies $(x(S), x(T)) \in C$.

We say that a feasible process $(\bar{x}, \bar{u})$ is a Pareto optimal process (or an efficient process) if there exists no other feasible process $(x, u)$ such that $g(x(S), x(T)) \lessgtr g(\bar{x}(S), \bar{x}(T))$.

We say that a feasible process $(\bar{x}, \bar{u})$ is a weak Pareto optimal process (or a weakly efficient process) if there exists no other feasible process $(x, u)$ such that $g(x(S), x(T))<$ $g(\bar{x}(S), \bar{x}(T))$.

\subsection{Hypotheses}

Let $(\bar{x}, \bar{u})$ be a reference feasible process of (MCP). We assume, throughout the paper, that, for some $\delta>0$, the following conditions are satisfied:

(H1) Function $f(\cdot, x, \cdot)$ is $\mathcal{L} \times \mathcal{B}^{m}$ measurable, for each $x \in \mathbb{R}^{n}$;

(H2) There exists an $\mathcal{L} \times \mathcal{B}$ measurable function $k:[S, T] \times \mathbb{R}^{m} \rightarrow \mathbb{R}$ such that $t \rightarrow$ $k(t, \bar{u}(t))$ is integrable, and for almost every $t \in[S, T]$,

$$
\left\|f(t, x, u)-f\left(t, x^{\prime}, u\right)\right\| \leq k(t, u)\left\|x-x^{\prime}\right\| \forall x, x^{\prime} \in \bar{x}(t)+\delta B, \quad \forall u \in U(t) ;
$$

( $B$ denotes the unit ball in $\mathbb{R}^{n}$.)

(H3) $\operatorname{Gr}(U)$ is $\mathcal{L} \times \mathcal{B}^{m}$ measurable;

(H4) $g$ is locally Lipschitz continuous.

\subsection{The maximum principle}

Let $\mathcal{H}:[S, T] \times \mathbb{R}^{n} \times \mathbb{R}^{n} \times \mathbb{R}^{m} \rightarrow \mathbb{R}$ denote the Unmaximized Hamiltonian function

$$
\mathcal{H}(t, x, p, u):=p \cdot f(t, x, u) .
$$

We now state the Pontryagin Maximum Principle.

Theorem 1 If $(\bar{x}, \bar{u})$ is a weak Pareto optimal process of $(M C P)$ and the function $t \rightarrow$ $k(t, \bar{u}(t))$ is integrable, then there exist a scalar $\lambda$ (equal to 0 or 1 ), a nonzero vector $\omega \in \mathbb{R}^{k}$, and an absolutely continuous function $p:[S, T] \rightarrow \mathbb{R}^{n}$ such that

$$
\begin{aligned}
& -\dot{p}(t) \in \operatorname{co} \partial_{x} \mathcal{H}(t, \bar{x}(t), p(t), \bar{u}(t)) \quad \text { a.e. } t \in[S, T], \\
& (p(S),-p(T)) \in \lambda \partial(\omega \cdot g)(\bar{x}(S), \bar{x}(T))+N_{C}(\bar{x}(S), \bar{x}(T)), \\
& \max _{u \in U(t)} \mathcal{H}(t, \bar{x}(t), p(t), u)=\mathcal{H}(t, \bar{x}(t), p(t), \bar{u}(t)) \quad \text { a.e. } t \in[S, T], \\
& \|p\|+\lambda>0,\|\omega\|=1, \omega \geq 0 .
\end{aligned}
$$

Proof (Sketch of the proof) In Bellaassali and Jourani [12], a maximum principle for a multiobjective dynamic optimization problem, where the dynamic is governed by a differential inclusion, is provided. The adjoint arc satisfies a differential inclusion involving the coderivative of the multifunction present in the dynamic of the problem. The transversality condition is stated in terms of the Mordukhovich's limiting subdifferential and of the limiting normal cone. 
The maximum principle for the scalar control problem (MCP) with $k=1$ is obtained in Vinter [58] as particular case of the extended Euler condition for an optimal control problem whose dynamic constraint is given by a differential inclusion.

By following the ideas in [58], the conditions above follow from [12, Corollary 3.1].

Observe that condition (1) can be equivalently stated as

$$
-\dot{p}(t)^{\top} \in p(t)^{\top} D_{x} f(t, \bar{x}(t), \bar{u}(t)) \quad \text { a.e. } t \in[S, T] .
$$

As it is easily verified, every Pareto optimal process is a weak Pareto optimal one, so that the maximum principle above is applicable to Pareto optimal solutions as well.

\subsection{More definitions}

When there exists $(\lambda, \omega, p)$ satisfying (1)-(4) we say that $(\bar{x}, \bar{u})$ is an extremal (or stationary) process of (MCP).

If $\lambda=1$, we say that $(\bar{x}, \bar{u})$ is a normal extremal process and (MCP) is said to be normal at $(\bar{x}, \bar{u})$.

We say that (MCP) is normal if it is normal at any extremal process $(\bar{x}, \bar{u})$, and we say that it is abnormal, otherwise. For example, optimal control problems with $C=C_{0} \times \mathbb{R}^{n}$, where $C_{0} \subset \mathbb{R}^{n}$ is closed, are normal.

In the smooth case, the concept of invexity involves the derivative of the defining functions of the problem with respect to all of the variables. As we are not going to assume any kind of differentiability of the defining functions of $(\mathrm{MCP})$ with respect to the control variables, we will make use of the following type of difference operator. Given $(t, x, u) \in[S, T] \times \mathbb{R}^{n} \times \mathbb{R}^{m}$, $(t, x, p, u) \in[S, T] \times \mathbb{R}^{n} \times \mathbb{R}^{n} \times \mathbb{R}^{m}$ and $\alpha \in(0,1)$ we define $\Delta_{\alpha} f(t, x, u), \Delta_{\alpha} \mathcal{H}(t, x, p, u)$ : $\mathbb{R}^{m} \rightarrow \mathbb{R}$, respectively, as

$$
\begin{aligned}
& \Delta_{\alpha} f(t, x, u)(\xi):=\frac{f(t, x, u+\alpha \xi)-f(t, x, u)}{\alpha}, \\
& \Delta_{\alpha} \mathcal{H}(t, x, p, u)(\xi):=\frac{\mathcal{H}(t, x, p, u+\alpha \xi)-\mathcal{H}(t, x, p, u)}{\alpha} .
\end{aligned}
$$

Vinter, in his book [58], has used a similar operator in order to "linearize" the dynamic system.

\section{MP-pseudoinvexity and sufficient optimality conditions}

In de Oliveira and Silva [27], we worked with mono-objective optimal control problems. A concept of generalized invexity was given. Here, such a concept is defined adequately for the multiobjective case. Similar results as in [27] are established.

\subsection{The normal case}

The concept of MP-pseudoinvexity was designed for optimal control problems using the same reasoning as that used to develop KT-invexity for mathematical programming problems (see Martin [41]). Let us recall that KT-invexity is to be applied for Kuhn-Tucker points, which are stationary points for which the Lagrange multiplier associated with the objective function is positive. Then it is natural that MP-pseudoinvexity is a sufficient optimality condition for a normal extremal process to be an optimal one. A more general definition of MP- 
pseudoinvexity is given in the next subsection, which is a sufficient optimality condition for every extremal process to be an optimal one.

We start the section with a technical lemma.

Lemma 1 Let $(\lambda, q) \in\{0,1\} \times W^{1,1}\left([S, T] ; \mathbb{R}^{n}\right)$ and $\alpha \in(0,1)$ be arbitrary. Let $(\bar{x}, \bar{u})$ be a feasible process of $(M C P)$ satisfying

$$
\Delta_{\alpha} \mathcal{H}(t, \bar{x}(t), q(t), \bar{u}(t), \lambda)(v) \leq 0 \quad \text { a.e. } t \in[S, T]
$$

for all $v \in \mathbb{R}^{m}$ such that $\bar{u}(t)+\alpha v \in U(t)$ a.e. $t \in[S, T]$. Then,

$$
\max _{u \in U(t)} \mathcal{H}(t, \bar{x}(t), q(t), u, \lambda)=\mathcal{H}(t, \bar{x}(t), q(t), \bar{u}(t), \lambda) \quad \text { a.e. } t \in[S, T]
$$

Proof Suppose on the contrary, that (5) does not hold. In this way, there exist $\epsilon>0$ and $A \subset[S, T]$ with positive measure such that

$$
\sup _{u \in U(t)} \mathcal{H}(t, \bar{x}(t), q(t), u, \lambda)-\epsilon>\mathcal{H}(t, \bar{x}(t), q(t), \bar{u}(t), \lambda) \quad \forall t \in A .
$$

Define the nonempty multifunction $U^{\prime}:[S, T] \rightrightarrows \mathbb{R}^{m}$ as

$$
U^{\prime}(t):=\{u \in U(t): \phi(t, u)>0\}
$$

where

$$
\phi(t, u)= \begin{cases}\mathcal{H}(t, \bar{x}(t), q(t), u, \lambda)-\epsilon-\mathcal{H}(t, \bar{x}(t), q(t), \bar{u}(t), \lambda), & \text { for } t \in A, \\ 1, & \text { for } t \in[S, T] \backslash A .\end{cases}
$$

Then

$$
\operatorname{Gr}\left(U^{\prime}\right)=\phi^{-1}((0, \infty)) \cap \operatorname{Gr}(U) .
$$

Provided (H1) and (H3) hold, we see that $\operatorname{Gr}\left(U^{\prime}\right)$ is $\mathcal{L} \times \mathcal{B}^{m}$ measurable. Applying Aumann's Measurable Selection Theorem (see Vinter [58, Theorem 2.3.12]) it follows that $U^{\prime}$ has a measurable selection, which in turn means that there exists a measurable function $\tilde{u}$ such that $\tilde{u}(t) \in U(t)$ a.e. $t \in[S, T]$ and

$$
\mathcal{H}(t, \bar{x}(t), q(t), \tilde{u}(t), \lambda)-\epsilon>\mathcal{H}(t, \bar{x}(t), q(t), \bar{u}(t), \lambda) \quad \text { a.e. } t \in A .
$$

Integrating, we obtain

$$
\int_{A}[\mathcal{H}(t, \bar{x}(t), q(t), \tilde{u}(t), \lambda)-\mathcal{H}(t, \bar{x}(t), q(t), \bar{u}(t), \lambda)] \mathrm{d} t>0 .
$$

Set

$$
v(t)= \begin{cases}\frac{\tilde{u}(t)-\bar{u}(t)}{\alpha}, & \text { for } t \in A, \\ 0, & \text { for } t \in[S, T] \backslash A .\end{cases}
$$

Then

$$
\bar{u}(t)+\alpha v(t)=\left\{\begin{array}{l}
\tilde{u}(t), \text { for } t \in A, \\
\bar{u}(t), \text { for } t \in[S, T] \backslash A,
\end{array}\right.
$$

so that $\bar{u}(t)+\alpha v(t) \in U(t)$ a.e. $t \in[S, T]$, and

$$
\Delta_{\alpha} \mathcal{H}(t, \bar{x}(t), q(t), \bar{u}(t), \lambda)(v(t))=\frac{\mathcal{H}(t, \bar{x}(t), q(t), \tilde{u}(t), \lambda)-\mathcal{H}(t, \bar{x}(t), q(t), \bar{u}(t), \lambda)}{\alpha},
$$


for $t \in A$, and

$$
\Delta_{\alpha} \mathcal{H}(t, \bar{x}(t), q(t), \bar{u}(t), \lambda)(v(t))=0
$$

otherwise. By hypothesis we have that

$$
\int_{S}^{T} \Delta_{\alpha} \mathcal{H}(t, \bar{x}(t), q(t), \bar{u}(t), \lambda)(v(t)) \mathrm{d} t \leq 0 .
$$

But then

$$
\begin{aligned}
0 & \geq \int_{S}^{T} \Delta_{\alpha} \mathcal{H}(t, \bar{x}(t), q(t), \bar{u}(t), \lambda)(v(t)) \mathrm{d} t \\
& =\int_{A} \Delta_{\alpha} \mathcal{H}(t, \bar{x}(t), q(t), \bar{u}(t), \lambda)(v(t)) \mathrm{d} t,
\end{aligned}
$$

which is in disagreement to (6).

Let $\alpha \in(0,1)$ be a scalar, $(\bar{x}, \bar{u})$ be a feasible process of (MCP) and $\zeta(t) \in$ $D_{x} f(t, \bar{x}(t), \bar{u}(t))$ a.e. $t \in[S, T]$. We define the set of " $\alpha$-feasible directions", denoted by $\mathcal{F}_{\alpha}(\bar{x}, \bar{u} ; \zeta)$, as the set of all pairs $(y, v)$ where $y:[S, T] \rightarrow \mathbb{R}^{n}, v:[S, T] \rightarrow \mathbb{R}^{m}$ are such that

$$
\begin{aligned}
& \dot{y}(t)=\zeta(t) y(t)+\Delta_{\alpha} f(t, \bar{x}(t), \bar{u}(t))(v(t)) \quad \text { a.e. } t \in[S, T], \\
& (y(S), y(T)) \in \bar{T}_{C}(\bar{x}(S), \bar{x}(T)), \\
& \bar{u}(t)+\alpha v(t) \in U(t) \quad \text { a.e. } t \in[S, T] .
\end{aligned}
$$

Observe that $\bar{u}(t)+\alpha v(t) \in U(t)$ a.e. $t \in[S, T]$ may be seen as a variation on the control variable and a solution of $\dot{y}(t)=\zeta(t) y(t)+\Delta_{\alpha} f(t, \bar{x}(t), \bar{u}(t))(v(t))$ a.e. $t \in[S, T]$ with $(y(S), y(T)) \in \bar{T}_{C}(\bar{x}(S), \bar{x}(T))$, as the corresponding variation on the state variable.

Before we give the definition of MP-pseudoinvexity, let us establish the following characterization of extremal processes of (MCP).

Proposition 1 Let $\alpha \in(0,1)$ and $(\bar{x}, \bar{u})$ be a feasible process of $(M C P)$. Assume that given

$$
\gamma=\left[\begin{array}{llll}
\gamma_{1} & \gamma_{2} & \ldots & \gamma_{k}
\end{array}\right]^{\top} \in D g(\bar{x}(S), \bar{x}(T))
$$

and

$$
\zeta(t) \in D_{x} f(t, \bar{x}(t), \bar{u}(t)) \quad \text { a.e. } t \in[S, T],
$$

for any pair $(y, v) \in \mathcal{F}_{\alpha}(\bar{x}, \bar{u} ; \zeta)$ one has

$$
\gamma_{j} \cdot(y(S), y(T)) \geq 0 \text { for at least one } j \in\{1,2, \ldots, k\} .
$$

Then $(\bar{x}, \bar{u})$ is an extremal process of $(M C P)$.

Proof Let us consider the auxiliary multiobjective control problem (ACP) below:

$$
\begin{aligned}
\text { minimize } & \phi(y(S), y(T))=\left[\gamma_{1} \gamma_{2} \ldots \gamma_{k}\right]^{\top}(y(S), y(T)) \\
\text { subject to } & \dot{y}(t)=\zeta(t) y(t)+\Delta_{\alpha} f(t, \bar{x}(t), \bar{u}(t))(v(t)) \quad \text { a.e. } t \in[S, T], \\
& (y(S), y(T)) \in K:=\bar{T}_{C}(\bar{x}(S), \bar{x}(T)), \\
& v(t) \in V(t):=\{v: \bar{u}(t)+\alpha v \in U(t)\} \quad \text { a.e. } t \in[S, T] .
\end{aligned}
$$


It is easy to see that $(\bar{y}, \bar{v})=(0,0)$ is a feasible process of $(\mathrm{ACP})$ with $\phi(\bar{y}(S), \bar{y}(T))=0$. Suppose that there exists a feasible process $(y, v)$ of (ACP) such that $\phi(y(S), y(T))<$ $\phi(\bar{y}(S), \bar{y}(T))$. In this case,

$$
\gamma_{j} \cdot(y(S), y(T))<\gamma_{j} \cdot(\bar{y}(S), \bar{y}(T))=0 \quad \forall j \in\{1,2, \ldots, k\},
$$

which contradicts the hypothesis. Therefore $(\bar{y}, \bar{v})$ is a weak Pareto optimal process of (ACP). By Theorem 1, there exist $(\lambda, \omega, p) \in\{0,1\} \times \mathbb{R}^{k} \times W^{1,1}\left([S, T] ; \mathbb{R}^{n}\right)$ such that

$$
\begin{aligned}
& -\dot{p}(t) \in \operatorname{co} \partial_{y}\left[p(t) \cdot\left[\zeta(t) \bar{y}(t)+\Delta_{\alpha} f(t, \bar{x}(t), \bar{u}(t))(\bar{v}(t))\right]\right] \quad \text { a.e. } t \in[S, T], \\
& (p(S),-p(T)) \in \lambda \partial(\omega \cdot \phi)(\bar{y}(S), \bar{y}(T))+N_{K}(\bar{y}(S), \bar{y}(T)), \\
& \max _{v \in V(t)}\left\{p(t) \cdot\left[\zeta(t) \bar{y}(t)+\Delta_{\alpha} f(t, \bar{x}(t), \bar{u}(t))(v)\right]\right\} \\
& \quad=p(t) \cdot\left[\zeta(t) \bar{y}(t)+\Delta_{\alpha} f(t, \bar{x}(t), \bar{u}(t))(\bar{v}(t))\right] \quad \text { a.e. } t \in[S, T], \\
& \|p\|+\lambda>0,\|\omega\|=1, \quad \omega \geq 0 .
\end{aligned}
$$

It follows from (7) that

$$
-\dot{p}(t)^{\top}=p(t)^{\top} \zeta(t) \in p(t)^{\top} D_{x} f(t, \bar{x}(t), \bar{u}(t)),
$$

so that

$$
-\dot{p}(t) \in \operatorname{co} \partial_{x} \mathcal{H}(t, \bar{x}(t), p(t), \bar{u}(t)) \quad \text { a.e. } t \in[S, T] .
$$

By (9) we see that $\max _{v \in V(t)}\left\{p(t) \cdot \Delta_{\alpha} f(t, \bar{x}(t), \bar{u}(t))(v)\right\}=0$ a.e. $t \in[S, T]$, that is, given $v \in \mathbb{R}^{m}$ with $\bar{u}(t)+\alpha v \in U(t)$ a.e. $t \in[S, T]$ one obtain

$$
\left.p(t) \cdot \Delta_{\alpha} f(t, \bar{x}(t), \bar{u}(t))(v)\right\} \leq 0 \quad \text { a.e. } t \in[S, T] .
$$

By applying Lemma 1 we get

$$
\max _{u \in U(t)} \mathcal{H}(t, \bar{x}(t), p(t), u)=\mathcal{H}(t, \bar{x}(t), p(t), \bar{u}(t)) \quad \text { a.e. } t \in[S, T] .
$$

It follows from (8) that

$$
(p(S),-p(T)) \in \lambda \omega^{\top}\left[\gamma_{1} \gamma_{2} \ldots \gamma_{k}\right]+N_{C}(\bar{x}(S), \bar{x}(T)) .
$$

But,

$$
\omega^{\top}\left[\begin{array}{llll}
\gamma_{1} & \gamma_{2} & \ldots & \gamma_{k}
\end{array}\right]^{\top} \in \omega^{\top} \operatorname{Dg}(\bar{x}(S), \bar{x}(T))
$$

so that

$$
(p(S),-p(T)) \in \lambda \partial(\omega \cdot g)(\bar{x}(S), \bar{x}(T))+N_{C}(\bar{x}(S), \bar{x}(T)) .
$$

Thus, from (10)-(13) we conclude that $(\bar{x}, \bar{u})$ is an extremal process of (ACP).

Definition 1 Let $(\bar{x}, \bar{u})$ be a feasible process. (MCP) is said to be MP-pseudoinvex at $(\bar{x}, \bar{u})$ if for every feasible process $(x, u)$ with $g(x(S), x(T)) \lesseqgtr g(\bar{x}(S), \bar{x}(T))$ and all $\alpha \in(0,1)$, given $\gamma=\left[\begin{array}{llll}\gamma_{1} & \gamma_{2} & \ldots & \gamma_{k}\end{array}\right]^{\top} \in D g(\bar{x}(S), \bar{x}(T))$ and $\zeta(t) \in D_{x} f(t, \bar{x}(t), \bar{u}(t))$ a.e. $t \in[S, T]$, there exist maps $(\eta, \xi) \in \mathcal{F}_{\alpha}(\bar{x}, \bar{u} ; \zeta)$ such that, for each $j \in\{1,2, \ldots, k\}$,

$$
\left\{\begin{array}{l}
g_{j}(x(S), x(T))=g_{j}(\bar{x}(S), \bar{x}(T)) \Rightarrow \gamma_{j} \cdot(\eta(S), \eta(T)) \leq 0 \\
\text { or } \\
g_{j}(x(S), x(T))<g_{j}(\bar{x}(S), \bar{x}(T)) \Rightarrow \gamma_{j} \cdot(\eta(S), \eta(T))<0 .
\end{array}\right.
$$

(MCP) is said to be MP-pseudoinvex if it is MP-pseudoinvex at every feasible process $(\bar{x}, \bar{u})$. 
We remark that in the definition above

$$
\begin{aligned}
& \eta=\eta(x, u, \bar{x}, \bar{u}), \\
& \xi=\xi(x, u, \bar{x}, \bar{u}) .
\end{aligned}
$$

When the problem data of (MCP) is continuously differentiable, definition above is equivalent to our previous definition of MP-pseudoinvexity given in de Oliveira et al. [22] for the smooth multiobjective case. Moreover, if $k=1$ the last definition agrees with MPpseudoinvexity stated in de Oliveira and Silva [27] for the nonsmooth (mono-objective) case.

The hypothesis below will be necessary in the sequel. Let $0 \neq w \in \mathbb{R}_{+}^{k}$. We say that hypothesis $(\mathrm{H}(w))$ is satisfied if

$$
\left\{j: g_{j}(x(S), x(T))<g_{j}(y(S), y(T))\right\} \cap\left\{j: w_{j}>0\right\} \neq \emptyset
$$

whenever $(x, u)$ and $(y, v)$ are feasible processes of (MCP) with $g(x(S), x(T)) \lessgtr$ $g(y(S), y(T))$.

Observe that hypothesis $(\mathrm{H}(w))$ is satisfied when $w>0$. It is also satisfied if $k=1$, that is, if the problem is mono-objective.

Theorem 2 Let $(\bar{x}, \bar{u})$ be a normal extremal process of $(M C P)$ with multipliers $(1, \omega, p)$. Assume that $(M C P)$ is MP-pseudoinvex at $(\bar{x}, \bar{u})$ and $(H(\omega))$ holds. Then $(\bar{x}, \bar{u})$ is a Pareto optimal process.

Proof Provided $(\bar{x}, \bar{u})$ is a normal extremal process with multipliers $(1, \omega, p)$, conditions (1)-(4) are verified. It follows that there exist $\gamma=\left[\gamma_{1} \gamma_{2} \ldots \gamma_{k}\right]^{\top} \in D g(\bar{x}(S), \bar{x}(T)), \zeta(t)$ $\in D_{x} f(t, \bar{x}(t), \bar{u}(t))$ a.e. $t \in[S, T]$ and $\left(v_{0}, v_{1}\right) \in N_{C}(\bar{x}(S), \bar{x}(T))$, obeying

$$
-\dot{p}(t)=\zeta(t)^{\top} p(t) \quad \text { a.e. } t \in[S, T]
$$

and

$$
(p(S),-p(T))=\omega^{\top} \gamma+\left(v_{0}, v_{1}\right) .
$$

Suppose that there does exist a feasible process $(x, u)$ of (MCP) such that $g(x(S), x(T))$ $\leq g(\bar{x}(S), \bar{x}(T))$.

By the MP-pseudoinvexity of (MCP), there exist maps $(\eta, \xi) \in \mathcal{F}_{\alpha}(\bar{x}, \bar{u} ; \zeta)$ satisfying (14). That is, in addition to (14), $(\eta, \xi)$ obeys

$$
\begin{aligned}
& \dot{\eta}(t)=\zeta(t) \eta(t)+\Delta_{\alpha} f(t, \bar{x}(t), \bar{u}(t))(\xi(t)) \quad \text { a.e. } t \in[S, T], \\
& \bar{u}(t)+\alpha \xi(t) \in U(t) \quad \text { a.e. } t \in[S, T],
\end{aligned}
$$

with

$$
(\eta(S), \eta(T)) \in \bar{T}_{C}(\bar{x}(S), \bar{x}(T)),
$$

for all $\alpha \in(0,1)$.

As $g(x(S), x(T)) \lessgtr g(\bar{x}(S), \bar{x}(T))$, by (14), given that $\omega \geq 0$ and $(\mathrm{H}(\omega))$ holds, one has

$$
\left[\omega^{\top} \gamma\right] \cdot(\eta(S), \eta(T))=\sum_{j=1}^{k} \omega_{j} \gamma_{j} \cdot(\eta(S), \eta(T))<0 .
$$

Hence, from (17),

$$
\left[\omega^{\top} \gamma\right] \cdot(\eta(S), \eta(T))+\int_{S}^{T} p(t) \cdot\left[\dot{\eta}(t)-\zeta(t) \eta(t)-\Delta_{\alpha} f(t, \bar{x}(t), \bar{u}(t))(\xi(t))\right] \mathrm{d} t<0 .
$$


On the other hand, by integration by parts, (15) and (16) one has

$$
\begin{aligned}
{\left[\omega^{\top} \gamma\right] \cdot(\eta(S), \eta(T))+\int_{S}^{T} p(t) \cdot\left[\dot{\eta}(t)-\zeta(t) \eta(t)-\Delta_{\alpha} f(t, \bar{x}(t), \bar{u}(t))(\xi(t))\right] \mathrm{d} t } \\
=\left[\omega^{\top} \gamma\right] \cdot(\eta(S), \eta(T))-(p(S),-p(T)) \cdot(\eta(S), \eta(T))-\int_{S}^{T} \dot{p}(t) \cdot \eta(t) \mathrm{d} t \\
\quad-\int_{S}^{T}\left[\zeta(t)^{\top} p(t) \cdot \eta(t)+p(t) \cdot \Delta_{\alpha} f(t, \bar{x}(t), \bar{u}(t))(\xi(t))\right] \mathrm{d} t \\
=-\left(v_{0}, v_{1}\right) \cdot(\eta(S), \eta(T))-\int_{S}^{T} \Delta_{\alpha} \mathcal{H}(t, \bar{x}(t), p(t), \bar{u}(t))(\xi(t)) \mathrm{d} t \geq 0,
\end{aligned}
$$

where the inequality, which follows from (19), the fact that $\left(v_{0}, v_{1}\right) \in N_{C}(\bar{x}(S), \bar{x}(T)),(18)$ and (3), contradicts (20).

In the case of weak Pareto optimality, hypothesis $(\mathrm{H}(\omega))$ is not required to be valid.

Theorem 3 Let $(\bar{x}, \bar{u})$ be a normal extremal process of $(M C P)$. Assume that $(M C P)$ is $M P$ pseudoinvex at $(\bar{x}, \bar{u})$. Then $(\bar{x}, \bar{u})$ is a weak Pareto optimal process.

Proof On the contrary, suppose that there exists another feasible process $(x, u)$ with $g(x(S), x(T))<g(\bar{x}(S), \bar{x}(T))$. Now proceed as in the proof of Theorem 2, observing that (20) holds without any additional assumption, since $g(x(S), X(T))<g(\bar{x}(S), \bar{x}(T))$ and $0 \neq \omega \geq 0$, to get a contradiction.

A variation of hypothesis $(\mathrm{H}(w))$ will be necessary. We say that hypothesis $(\mathrm{H})$ is satisfied if, given a normal extremal process of (MCP) with multipliers $(1, w, p),(\mathrm{H}(w))$ is verified.

As immediate consequences of the last results we have the following two theorems. Next, we establish the converse of Theorem 4 below.

Theorem 4 Assume that (MCP) is MP-pseudoinvex and $(H)$ holds. Then every normal extremal process is a Pareto optimal process.

Theorem 5 Assume that (MCP) is MP-pseudoinvex. Then every normal extremal process is a weak Pareto optimal process.

In [22], weak Pareto optimality was regarded. The last result generalizes, for the nonsmooth case, the corresponding one in [22].

Theorem 6 Assume that $(M C P)$ is such that every extremal process is a Pareto optimal process. Then it is MP-pseudoinvex.

Proof On the contrary, assume that (MCP) is not MP-pseudoinvex. Then there exist feasible processes $(x, u),(\bar{x}, \bar{u})$ with $g(x(S), x(T)) \lesseqgtr g(\bar{x}(S), \bar{x}(T)), \alpha \in(0,1), \gamma=$ $\left[\gamma_{1} \gamma_{2} \ldots \gamma_{k}\right]^{\top} \in D g(\bar{x}(S), \bar{x}(T))$ and $\zeta(t) \in D_{x} f(t, \bar{x}(t), \bar{u}(t))$ a.e. $t \in[S, T]$, such that for every pair $(\eta, \xi) \in \mathcal{F}_{\alpha}(\bar{x}, \bar{u} ; \zeta)$ one has for at least one $j \in\{1,2, \ldots, k\}$ that

$$
\left\{\begin{array}{l}
\gamma_{j} \cdot(\eta(S), \eta(T)) \geq 0 \text { with } g_{j}(x(S), x(T))<g_{j}(\bar{x}(S), \bar{x}(T)), \\
\text { or } \\
\gamma_{j} \cdot(\eta(S), \eta(T))>0 \text { with } g_{j}(x(S), x(T))=g_{j}(\bar{x}(S), \bar{x}(T)) .
\end{array}\right.
$$

The assumptions of Proposition 1 are then fulfilled. Therefore $(\bar{x}, \bar{u})$ is an extremal process. By hypothesis it is a Pareto optimal process, contradicting the fact that $g(x(S), x(T)) \lessgtr$ $g(\bar{x}(S), \bar{x}(T))$ for a feasible process $(x, u)$. Thus (MCP) is MP-pseudoinvex. 
Corollary 1 Assume that $(M C P)$ is normal and that $(H)$ holds. Then $(M C P)$ is $M P$ pseudoinvex if, and only if, every extremal process is a Pareto optimal process.

Proof It follows directly from Theorems 2 and 6.

Once Definition 1 is suitable for Pareto optimality, the converse of Theorem 5 is not valid, so that a similar result as Corollary 1 for weak Pareto optimality does not hold. However, if condition $g(x(S), x(T)) \lesseqgtr g(\bar{x}(S), \bar{x}(T))$ is changed to $g(x(S), x(T))<g(x(S), x(T))$ in Definition 1 , then the converse of Theorem 5 as well as a similar result like Corollary 1 are valid.

In the sequel, a simple non-convex problem is presented.

Example 1 Let us consider the following bi-objective optimal control problem:

$$
\begin{aligned}
\text { minimize } & g(x(0), x(1))=\left((x(1)-2)^{2}, \sqrt{x(1)+1}\right) \\
\text { subject to } \dot{x}(t)=u(t) \quad \text { a.e. } t \in[0,1], & \\
& (x(0), x(1)) \in C=\{0\} \times \mathbb{R}, \\
& u(t) \in U(t)=[0,4] \quad \text { a.e. } t \in[0,1] .
\end{aligned}
$$

It is easily seen that the problem is normal and that

$-(\bar{x}, \bar{u})=(0,0)$ is an extremal process with $\bar{\lambda}=1, \bar{w}=\left(\frac{1}{2}, \frac{1}{2}\right)$ and $\bar{p}(t) \equiv \frac{7}{4}$;

- $(\tilde{x}, \tilde{u})$ such that $\tilde{x}(1) \in(0,2)$ are extremal processes with $\tilde{\lambda}=1, \tilde{p}(t) \equiv 0$ and $\tilde{w}=$ $\left(1-w_{2}, w_{2}\right), w_{2}=\frac{4(\tilde{x}(1)-2) \sqrt{\tilde{x}(1)+1}}{4(\tilde{x}(1)-2) \sqrt{\tilde{x}(1)+1}-1} \in(0,1) ;$

- $(\hat{x}, \hat{u})$ such that $\hat{x}(1)=2$ are extremal processes with $\hat{\lambda}=1, \hat{p}(t) \equiv 0$ and $\hat{w}=(1,0)$.

Note that hypothesis $(\mathrm{H}(w))$ is satisfied for extremals $(\bar{x}, \bar{u})$ and $(\tilde{x}, \tilde{u})$, but it is not verified for extremals $(\hat{x}, \hat{u})$.

The problem is MP-pseudoinvex. Indeed, let $(\bar{x}, \bar{u})$ be a feasible process and $\alpha \in(0,1)$. The set of $\alpha$-feasible directions is given by

$$
\mathcal{F}_{\alpha}(\bar{x}, \bar{u})=\left\{y, v:[0,1] \rightarrow \mathbb{R} / y(t)=\int_{0}^{t} v(s) \mathrm{d} s, \bar{u}(t)+\alpha v(t) \in[0,4] \text { a.e. } t\right\} .
$$

Let $(x, u)$ be a feasible process of the problem above with $g(x(0), x(1)) \lesseqgtr g(\bar{x}(0), \bar{x}(1))$, so that

$$
(x(1)-2)^{2}<(\bar{x}(1)-2)^{2} \text { and } \sqrt{x(1)+1}<\sqrt{\bar{x}(1)+1}
$$

or

$$
(x(1)-2)^{2}=(\bar{x}(1)-2)^{2} \text { and } \sqrt{x(1)+1}<\sqrt{\bar{x}(1)+1} .
$$

In both cases one has $\bar{x}(1)>2$. One has also that $D g(\bar{x}(0), \bar{x}(1))=\left[\begin{array}{ll}\gamma_{1} & \gamma_{2}\end{array}\right]^{\top}$ with

$$
\gamma_{1}=(0,2(\bar{x}(1)-2)) \text { and } \gamma_{2}=\left(0,2^{-1}(x(1)+1)^{-1 / 2}\right) \text {. }
$$

Let us take $\eta(t)=-\bar{x}(t)$ and $\xi(t)=-\bar{u}(t)$ a.e. $t \in[0,1]$. Clearly, $(\eta, \xi) \in \mathcal{F}_{\alpha}(\bar{x}, \bar{u})$. Furthermore,

$$
\gamma_{1} \cdot(\eta(0), \eta(1))=-2(\bar{x}(1)-2) \bar{x}(1)<0
$$

and

$$
\gamma_{2} \cdot(\eta(0), \eta(1))=-\frac{\bar{x}(1)}{2 \sqrt{\bar{x}(1)+1}}<0 .
$$


Therefore, the problem is MP-pseudoinvex, as claimed. Hence, by Theorem 2, the extremals $(\bar{x}, \bar{u})$ and $(\tilde{x}, \tilde{u})$ are Pareto optimal processes. By Theorem 3, all extremals are weak Pareto optimal processes.

Observe that a complete characterization of the weak Pareto optimal processes is obtained, while the characterization of the Pareto optimal processes was not possible, once hypothesis $(\mathrm{H})$ is not satisfied.

\subsection{The general case}

MP-pseudoinvexity for the general case is defined here. We show that MP-pseudo-invexity is a sufficient condition for extremal processes to be optimal processes; and we prove that optimal control problems such that all extremal processes are optimal necessarily obey the MP-pseudoinvexity conditions.

We demonstrate that, under problem normality, MP-pseudoinvexity defined in this section and Definition 1 are equivalent.

Before the new definition of MP-pseudoinvexity, let us introduce the following set of multipliers and state a proposition in the same lines as Proposition 1 in the last section.

Let $(\bar{x}, \bar{u})$ be a feasible process of (MCP). We define

$$
\begin{aligned}
\Lambda(\bar{x}, \bar{u}):= & \left\{(\lambda, \omega, p) \in\{0,1\} \times \mathbb{R}^{k} \times W^{1,1}\left([S, T] ; \mathbb{R}^{n}\right)\right. \text { such that } \\
& -\dot{p}(t)=\zeta(t)^{\top} p(t), \zeta(t) \in D_{x} f(t, \bar{x}(t), \bar{u}(t)) \quad \text { a.e. } t \in[S, T] ; \\
& (p(S),-p(T))=\lambda \omega^{\top} \gamma+\left(v_{0}, v_{1}\right), \\
& \gamma=\left[\gamma_{1} \gamma_{2} \ldots \gamma_{k}\right]^{\top} \in D g(\bar{x}(S), \bar{x}(T)),\left(v_{0}, v_{1}\right) \in N_{C}(\bar{x}(S), \bar{x}(T)) ; \\
& \|p\|+\lambda>0,\|\omega\|=1, \omega \geq 0\} .
\end{aligned}
$$

Let $\alpha \in(0,1)$. Given $(\lambda, \omega, p) \in \Lambda(\bar{x}, \bar{u})$ and maps $y:[S, T] \rightarrow \mathbb{R}^{n}$ and $v:[S, T] \rightarrow \mathbb{R}^{m}$, we denote

$$
\begin{aligned}
\mathcal{J}_{\alpha}(\bar{x}, \bar{u} ; \lambda, \omega, p)(y, v):= & \lambda\left[\omega^{\top} \gamma\right] \cdot(y(S), y(T)) \\
& +\int_{S}^{T} p(t) \cdot\left[\dot{y}(t)-\zeta(t) y(t)-\Delta_{\alpha} f(t, \bar{x}(t), \bar{u}(t))(v(t))\right] d t .
\end{aligned}
$$

Proposition 2 Let $\alpha \in(0,1)$ and $(\bar{x}, \bar{u})$ be a feasible process of $(M C P)$. If given $(\lambda, \omega, p) \in$ $\Lambda(\bar{x}, \bar{u})$ one has

$$
\mathcal{J}_{\alpha}(\bar{x}, \bar{u} ; \lambda, \omega, p)(y, v) \geq 0
$$

for all $(y, v)$ such that

$$
\begin{aligned}
& (y(S), y(T)) \in \bar{T}_{C}(\bar{x}(S), \bar{x}(T)), \\
& \bar{u}(t)+\alpha v(t) \in U(t) \quad \text { a.e. } t \in[S, T],
\end{aligned}
$$

then $(\bar{x}, \bar{u})$ is an extremal process of $(M C P)$.

Proof Let $(\lambda, \omega, p) \in \Lambda(\bar{x}, \bar{u})$. It is enough to show that

$$
\max _{u \in U(t)} \mathcal{H}(t, \bar{x}(t), p(t), u)=\mathcal{H}(t, \bar{x}(t), p(t), \bar{u}(t)) \quad \text { a.e. } t \in[S, T] .
$$

Assume that this fact is not true. Take $y \equiv 0$. The demonstration follows now as in the proof of Lemma 1, where a contradiction is gotten. 
Remark 1 If $(\lambda, \omega, p) \in \Lambda(\bar{x}, \bar{u})$ has $\lambda=1$ in Proposition 2 , it is easily verified from the proof of the proposition that $(\bar{x}, \bar{u})$ is a normal extremal process.

Definition 2 Let $(\bar{x}, \bar{u})$ be a feasible process. (MCP) is said to be MP-pseudoinvex at $(\bar{x}, \bar{u})$ if given $\alpha \in(0,1)$ and $(\lambda, \omega, p) \in \Lambda(\bar{x}, \bar{u})$, there exist maps $\eta:[S, T] \rightarrow \mathbb{R}^{n}$ and $\xi:[S, T] \rightarrow \mathbb{R}^{m}$ such that

$$
g(x(S), x(T)) \lesseqgtr g(\bar{x}(S), \bar{x}(T)) \Rightarrow \mathcal{J}_{\alpha}(\bar{x}, \bar{u} ; \lambda, \omega, p)(\eta, \xi)<0
$$

for all feasible processes $(x, u)$, where

$$
\begin{aligned}
& (\eta(S), \eta(T)) \in \bar{T}_{C}(\bar{x}(S), \bar{x}(T)), \\
& \bar{u}(t)+\alpha \xi(t) \in U(t) \quad \text { a.e. } t \in[S, T] .
\end{aligned}
$$

When conditions above hold true only for multipliers $(\lambda, \omega, p) \in \Lambda(\bar{x}, \bar{u})$ with $\lambda=1$, (MCP) is said to be normal MP-pseudoinvex at $(\bar{x}, \bar{u})$.

(MCP) is said to be (normal) MP-pseudoinvex if it is (normal) MP-pseudoinvex at every feasible process.

Theorem 7 Let $(\bar{x}, \bar{u})$ be a (normal) extremal process of $(M C P)$. Suppose that $(M C P)$ is (normal) MP-pseudoinvex at $(\bar{x}, \bar{u})$. Then $(\bar{x}, \bar{u})$ is a Pareto optimal process.

Proof As $(\bar{x}, \bar{u})$ is extremal, there exists $(\lambda, \omega, p) \in \Lambda(\bar{x}, \bar{u})$ satisfying

$$
\max _{u \in U(t)} \mathcal{H}(t, \bar{x}(t), p(t), u)=\mathcal{H}(t, \bar{x}(t), p(t), \bar{u}(t)) \quad \text { a.e. } t \in[S, T] .
$$

Suppose that there does exist a feasible process $(x, u)$ of (MCP) such that $g(x(S), x(T)) \lessgtr$ $g(\bar{x}(S), \bar{x}(T))$. Let $\alpha \in(0,1)$. By the MP-pseudoinvexity of (MCP), there exist maps $\eta$ and $\xi$ verifying (21)-(23). However, as in the proof of Theorem 2,

$$
\begin{aligned}
\mathcal{J}_{\alpha}(\bar{x}, \bar{u} ; \lambda, \omega, p)(\eta, \xi)= & \lambda\left[\omega^{\top} \gamma\right] \cdot(\eta(S), \eta(T)) \\
& +\int_{S}^{T} p(t) \cdot\left[\dot{\eta}(t)-\zeta(t) \eta(t)-\Delta_{\alpha} f(t, \bar{x}(t), \bar{u}(t))(\xi(t))\right] \mathrm{d} t \\
= & -\left(v_{0}, v_{1}\right) \cdot(\eta(S), \eta(T)) \\
& -\int_{S}^{T} \Delta_{\alpha} \mathcal{H}(t, \bar{x}(t), p(t), \bar{u}(t))(\xi(t)) \mathrm{d} t \geq 0,
\end{aligned}
$$

which contradicts (21).

Corollary 2 Let $(\bar{x}, \bar{u})$ be a (normal) extremal process of $(M C P)$. Suppose that $(M C P)$ is (normal) MP-pseudoinvex at $(\bar{x}, \bar{u})$. Then $(\bar{x}, \bar{u})$ is a weak Pareto optimal process.

Proof It follows directly from Theorem 8 by noting that every Pareto process is a weak Pareto process.

The two results in the sequel are obtained from Theorem 7.

Theorem 8 Suppose that (MCP) is (normal) MP-pseudoinvex. Then every (normal) extremal process is a Pareto optimal process.

Corollary 3 Suppose that (MCP) is (normal) MP-pseudoinvex. Then every (normal) extremal process is a weak Pareto optimal process. 
The converse of Corollary 3 is not valid, unless in Definition 2 condition $g(x(S), x(T)) \lessgtr$ $g(\bar{x}(S), \bar{x}(T))$ is replaced by $g(x(S), x(T))<g(\bar{x}(S), \bar{x}(T))$. The converse of Theorem 7 is true.

Theorem 9 Suppose that $(M C P)$ is such that every (normal) extremal process is a Pareto optimal process. Then it is (normal) MP-pseudoinvex.

Proof Let $(x, u),(\bar{x}, \bar{u})$ be feasible processes with $g(x(S), x(T)) \lesseqgtr g(\bar{x}(S), \bar{x}(T))$, $\alpha \in(0,1)$ and $(\lambda, \omega, p) \in \Lambda(\bar{x}, \bar{u})$. If for all $(\eta, \xi)$ such that (22)-(23) hold one has $\mathcal{J}_{\alpha}(\bar{x}, \bar{u} ; \lambda, \omega, p)(\eta, \xi) \geq 0$, by Proposition $2,(\bar{x}, \bar{u})$ is an extremal process, which, by assumption, is Pareto optimal. But this contradicts the fact that $g(x(S), x(T)) \lessgtr$ $g(\bar{x}(S), \bar{x}(T))$. Therefore there exists $(\eta, \xi)$ such that (21)-(23) are verified.

Corollary 4 (MCP) is (normal) MP-pseudoinvex if, and only if, every (normal) extremal process is a Pareto optimal process.

Proof It is an immediate consequence of Theorems 8 and 9.

Corollary 5 Assume that $(H)$ is valid. If $(M C P)$ is MP-pseudoinvex according to Definition 1, then it is normal MP-pseudoinvex (according to Definition 2).

Proof It follows directly from Theorems 4 and 9.

Corollary 6 If $(M C P)$ is MP-pseudoinvex according to Definition 2, then it is so according to Definition 1.

Proof It can be verified directly from Theorems 8 and 6 .

Corollary 7 If $(M C P)$ is normal and $(H)$ is valid, then the two definitions of MPpseudoinvexity are equivalent.

Proof It is a straightforward consequence of Corollary 1, Theorem 9 and Corollary 6.

It is clear from the proof of Theorem 7 that condition (21) in the Definition 2 may be changed to

$$
\begin{aligned}
g(x(S), x(T)) \lessgtr g(\bar{x}(S), \bar{x}(T)) \Rightarrow & \left(\nu_{0}, v_{1}\right) \cdot(\eta(S), \eta(T)) \\
& +\int_{S}^{T} \Delta_{\alpha} \mathcal{H}(t, \bar{x}(t), p(t), \bar{u}(t))(\xi(t)) \mathrm{d} t>0,
\end{aligned}
$$

in such a way that Theorems 7,8 and 9 are still valid. Naturally, the corresponding change in the assumptions of Proposition 2 should be made. The resulting modified definition of MP-pseudoinvexity is the direct generalization of MP-pseudoinvexity given in [27].

We now revisit Example 1.

Example 2 Let us consider the same bi-objective optimal control problem from Example 1:

$$
\begin{aligned}
\text { minimize } & g(x(0), x(1))=\left((x(1)-2)^{2}, \sqrt{x(1)+1}\right) \\
\text { subject to } & \dot{x}(t)=u(t) \text { a.e. } t \in[0,1], \\
& (x(0), x(1)) \in C=\{0\} \times \mathbb{R}, \\
& u(t) \in U(t)=[0,4] \quad \text { a.e. } t \in[0,1] .
\end{aligned}
$$

As we saw, the problem is normal. The problem is MP-pseudoinvex as stated by Definition 2. Indeed, let $(\bar{x}, \bar{u})$ be a feasible process. Then

$$
\Lambda(\bar{x}, \bar{u})=\left\{(\lambda, \omega, p): \lambda=1, \omega_{1}+\omega_{2}=1, \omega_{1}, \omega_{2} \geq 0,\right.
$$




$$
\left.p(t) \equiv-2 \omega_{1}(\bar{x}(1)-2)-2^{-1} \omega_{2}(\bar{x}(1)+1)^{-1 / 2}\right\} .
$$

Let $\alpha \in(0,1),(\lambda, \omega, p) \in \Lambda(\bar{x}, \bar{u})$ and $(x, u)$ be a feasible process of the problem above with $g(x(0), x(1)) \lesseqgtr g(\bar{x}(0), \bar{x}(1))$, so that

$$
(x(1)-2)^{2}<(\bar{x}(1)-2)^{2} \text { and } \sqrt{x(1)+1}<\sqrt{\bar{x}(1)+1}
$$

or

$$
(x(1)-2)^{2}=(\bar{x}(1)-2)^{2} \text { and } \sqrt{x(1)+1}<\sqrt{\bar{x}(1)+1} .
$$

In both cases one has $\bar{x}(1)>2$. Let us take $\eta(t)=-\bar{x}(t)$ and $\xi(t)=-\bar{u}(t)$ a.e. $t \in[0,1]$. Clearly, $(\eta(0), \eta(1)) \in\{0\} \times \mathbb{R}=\bar{T}_{C}(\bar{x}(0), \bar{x}(1))$ and $\bar{u}(t)+\alpha \xi(t) \in[0,4]=U(t)$ a.e. $t \in$ $[S, T]$. Furthermore,

$$
\mathcal{J}_{\alpha}(\bar{x}, \bar{u} ; \lambda, \omega, p)(\eta, \xi)=-2 \omega_{1}(\bar{x}(1)-2) \bar{x}(1)-2^{-1} \omega_{2}(\bar{x}(1)+1)^{-1 / 2} \bar{x}(1)<0 .
$$

Therefore, the problem is MP-pseudoinvex, as claimed. Thus, by Theorem 8, every extremal process is a Pareto optimal process.

Here, a complete characterization of the Pareto optimal processes is obtained. This was not the case in Example 1.

The next example is a slight adaptation of Exercise 22.4 in Clarke [18].

Example 3 Let us consider the following bi-objective optimal control problem:

$$
\begin{aligned}
\text { minimize } & g(x(0), y(0), x(1), y(1))=\left(g_{1}(y(1)), g_{2}(y(1))\right) \\
\text { subject to } & (\dot{x}(t), \dot{y}(t))=(u(t), v(t)) \text { a.e. } t \in[0,1] \\
& (x(0), y(0), x(1), y(1)) \in C=\{0\} \times\{0\} \times\{1\} \times \mathbb{R}, \\
& (u(t), v(t)) \in U(t)=\left\{(u, v): u^{2}+v^{2} \leq 1\right\} \quad \text { a.e. } t \in[0,1] .
\end{aligned}
$$

Assume that $g_{i}$ are such that $g_{i}^{\prime}(0)>0, i=1,2$.

It is easy to see that $(x(t), y(t), u(t), v(t))=(t, 0,1,0)$ a.e. $t \in[0,1]$ is the only feasible process, so that it is optimal.

Simple calculations show that $(x(t), y(t), u(t), v(t))=(t, 0,1,0)$ a.e. $t \in[0,1]$ is the only extremal process, with $\lambda=0, \omega_{1}, \omega_{2} \geq 0, \omega_{1}+\omega_{2}=1$, and $(p(t), q(t))=(c, 0)$ a.e. $t \in[0,1]$, where $c<0$.

Therefore, one has that every extremal process is an optimal process. By Theorem 9, the problem is MP-pseudoinvex.

Note that this problem does not admit any normal extremal process, that is, extremals with $\lambda=1$.

The example below is similar to the last one, but admits both normal and abnormal extremal processes. This is due to a simple modification in the set of admissible control functions. As the main goal is to illustrate the occurrence of normal and abnormal extremals in the same problem, for simplicity, we consider the mono-objective case.

Example 4 Let us consider the following optimal control problem:

$$
\begin{aligned}
\text { minimize } & g(x(0), y(0), x(1), y(1))=g_{1}(y(1)) \\
\text { subject to } & (\dot{x}(t), \dot{y}(t))=(u(t), v(t)) \text { a.e. } t \in[0,1], \\
& (x(0), y(0), x(1), y(1)) \in C=\{0\} \times\{0\} \times\{1\} \times \mathbb{R}, \\
& (u(t), v(t)) \in U(t)=[0,1] \times[0,1] \text { a.e. } t \in[0,1] .
\end{aligned}
$$


Assume that $g_{1}$ is such that $g_{1}^{\prime}(y)>0$ for all $y$. Again, $(x(t), y(t), u(t), v(t))=(t, 0,1,0)$ a.e. $t \in[0,1]$ is the only feasible process, so that it is optimal.

In this case, the extremal processes are given by:

$-(\bar{x}, \bar{y}, \bar{u}, \bar{v})=(t, 0,1,0)$ with $\lambda=1$ and $(p(t), q(t))=\left(\bar{c},-g_{1}^{\prime}(y(1))\right)$ a.e. $t \in[0,1]$, where $\bar{c} \geq 0$;

$-(\tilde{x}, \tilde{y}, \tilde{u}, \tilde{v})=(t, \tilde{y}, 1, \tilde{v})$ with $\lambda=0$ and $(p(t), q(t))=(\tilde{c}, 0)$ a.e. $t \in[0,1]$, where $\tilde{c}>0, \tilde{y}(t)=\int_{0}^{t} \tilde{v}(s) \mathrm{d} s, \tilde{v}(t)=\tilde{v} \in[0,1]$ a.e. $t \in[0,1]$.

Thence, every normal extremal process is an optimal process, but not all extremal processes are optimal. By Corollary 4, the problem is not MP-pseudoinvex according to Definition 2. Nevertheless, it is MP-pseudoinvex according to Definition 1. Indeed, let $\alpha \in(0,1)$ and $(\bar{x}, \bar{y}, \bar{u}, \bar{v})$ be a feasible process. The set of $\alpha$-feasible directions is given as

$$
\begin{aligned}
\mathcal{F}_{\alpha}(\bar{x}, \bar{y}, \bar{u}, \bar{v})= & \left\{\eta_{1}, \eta_{2}, \xi_{1}, \xi_{2}:[0,1] \rightarrow \mathbb{R} / \eta_{i}(t)=\int_{0}^{t} \xi_{i}(s) \mathrm{d} s, i=1,2,\right. \\
& \eta_{1}(0)=\eta_{2}(0)=0, \eta_{1}(1)=0, \bar{u}(t)+\alpha \xi_{1}(t) \in[0,1], \\
& \left.\bar{v}(t)+\alpha \xi_{2}(t) \in[0,1] \text { a.e. } t\right\} .
\end{aligned}
$$

Let $(x, y, u, v)$ be a feasible process of the problem above with $g_{1}(y(1))<g_{1}(\bar{y}(1))$, so that $y(1)<\bar{y}(1)$ and $\bar{y}(1)>0$. Define

$$
\eta_{1}(t)=x(t)-\bar{x}(t) \text { and } \xi_{1}(t)=u(t)-\bar{u}(t) \quad \text { a.e. } t \in[0,1],
$$

and

$$
\eta_{2}(t)=-\bar{y}(t) \text { and } \xi_{2}(t)=-\bar{v}(t) \text { a.e. } t \in[0,1] .
$$

Then, $\left(\eta_{1}, \eta_{2}, \xi_{1}, \xi_{2}\right) \in \mathcal{F}_{\alpha}(\bar{x}, \bar{y}, \bar{u}, \bar{v})$ and

$$
\nabla g(\bar{x}(0), \bar{y}(0), \bar{x}(1), \bar{y}(1)) \cdot\left(\eta_{1}(0), \eta_{2}(0), \eta_{1}(1), \eta_{2}(1)\right)=g_{1}^{\prime}(\bar{y}(1)) \eta_{2}(1)<0 .
$$

Thus, the problem is MP-pseudoinvex according to Definition 1, as claimed.

Analyzing Examples 1 and 2, one can say that Definition 2 is stronger than Definition 1.

Let us make some more comments in order to distinguish these definitions. Consider a problem where there are only abnormal extremal processes (like Example 3 above). Even if the problem is MP-pseudoinvex according to Definition 1, Theorems 2-5 are not applicable (they are only applicable to normal processes), so that it is not possible to characterize the optimal processes in this case. However, if the problem is MP-pseudoinvex according to Definition 2, Theorems 7 and 8 can be applied.

Another situation is when the problem possesses both normal and abnormal extremals (see Example 4). Again, the results related with MP-pseudoinvexity according to Definition 1 are not useful and a complete characterization of all of the optimal processes is not achieved. But this is possible by making use of Definition 2 and the results in this section.

Observe that if (MCP) is MP-pseudoinvex according to Definition 2, inequality (21) should be satisfied for all $(\lambda, \omega, p) \in \Lambda(\bar{x}, \bar{u})$, including both cases with $\lambda=0$ and $\lambda=1$.

The case with $\lambda=0$ is reduced to the following. If $g(x(S), x(T)) \lessgtr g(\bar{x}(S), \bar{x}(T))$, then, along with (22)-(23), one has to have

$$
\mathcal{J}_{\alpha}(\bar{x}, \bar{u} ; 0, \omega, p)(\eta, \xi)=\int_{S}^{T} p(t) \cdot\left[\dot{\eta}(t)-\zeta(t) \eta(t)-\Delta_{\alpha} f(t, \bar{x}(t), \bar{u}(t))(\xi(t))\right] d t<0
$$


for all $p \in W^{1,1}\left([S, T] ; \mathbb{R}^{n}\right)$ such that

$$
-\dot{p}(t)=\zeta(t)^{\top} p(t), \text { for some } \zeta(t) \in D_{x} f(t, \bar{x}(t), \bar{u}(t)) \quad \text { a.e. } t \in[S, T],
$$

with $(p(S),-p(T)) \in N_{C}(\bar{x}(S), \bar{x}(T))$ and $\|p\|>0$.

Let us consider the case with $\lambda=1$. A sufficient condition for the validity of (21)-(23) is the MP-pseudoinvexity of (MCP) according to Definition 1 along with hypothesis (H). Indeed, see Corollary 5 (or directly from the definitions). In addition, if (MCP) is normal and (H) holds true, the case $\lambda=1$ (normal MP-pseudoinvexity) is equivalent to MP-pseudoinvexity according to Definition 1 (see Corollaries 1 and 4).

In Corollary 6 it is showed that MP-pseudoinvexity according to Definition 2 implies in MP-pseudoinvexity according to Definition 1 . But the converse may not be true, unless the problem is normal and $(\mathrm{H})$ is valid. Thus, MP-pseudoinvexity according to Definition 2 is stronger than according to Definition 1.

Summarizing, if Definition 1 and $(\mathrm{H})$ are satisfied, then the case $\lambda=1$ is achieved. In order to (MCP) to be MP-pseudoinvex according to Definition 2, the case $\lambda=0$ has to be achieved as well.

\section{Generalized invexity and scalarization}

Let $w=\left(w_{1}, w_{2}, \ldots, w_{k}\right) \in \mathbb{R}_{+}^{k}$ be a nonzero vector. We will study the relationship between $(\mathrm{MCP})$ and the following weighting scalar problem $(\mathrm{P}(w))$ :

$$
\begin{aligned}
\text { minimize } & g_{0}(x(S), x(T)) \\
\text { subject to } & \dot{x}(t)=f(t, x(t), u(t)) \quad \text { a.e. } t \in[S, T], \\
& (x(S), x(T)) \in C, \\
& u(t) \in U(t) \quad \text { a.e. } t \in[S, T],
\end{aligned}
$$

where $g_{0}: \mathbb{R}^{n} \times \mathbb{R}^{n} \rightarrow \mathbb{R}$ is given by

$$
g_{0}(x, y)=w \cdot g(x, y)=\sum_{j=1}^{k} w_{j} g_{j}(x, y) .
$$

A feasible process of $(\mathrm{P}(w))$ is exactly the same as a feasible process of (MCP).

Given $0 \neq w \in \mathbb{R}_{+}^{k}$, a feasible process $(\bar{x}, \bar{u})$ is said to be an optimal process of $(\mathrm{P}(w))$ if it is a global optimal solution of the problem, that is, $g_{0}(x(S), x(T)) \geq g_{0}(\bar{x}(S), \bar{x}(T))$ for all feasible processes $(x, u)$.

The study in this section will be carried out through invexity assumptions. We first make use of MP-pseudoinvexity (Definition 2). Afterwards, we define the notion MP-invexity and perform the same kind of study.

Theorem 10 Let $0 \neq w \in \mathbb{R}_{+}^{k}$ and assume that $(H(w))$ is satisfied. If $(\bar{x}, \bar{u})$ is an optimal process of $(P(w))$, then it is a Pareto optimal process of $(M C P)$.

Proof Suppose that there exists a feasible process $(x, u)$ of (MCP) such that $g(x(S), x(T)) \lessgtr$ $g(\bar{x}(S), \bar{x}(T))$. As $(\mathrm{H}(w))$ is satisfied, it follows that $w \cdot g(x(S), x(T))<w \cdot g(\bar{x}(S), \bar{x}(T))$, which contradicts the global optimality of $(\bar{x}, \bar{u})$ in $(\mathrm{P}(w))$.

When $0<w \in \mathbb{R}^{k}$, then $(\mathrm{H}(w))$ is automatically satisfied. In this case, if $(\bar{x}, \bar{u})$ is an optimal process of $(\mathrm{P}(w))$, by Theorem 10, it is a Pareto optimal process of (MCP).

Hypothesis $(\mathrm{H}(w))$ is unnecessary in the case of weak Pareto optimality. 
Theorem 11 Let $0 \neq w \in \mathbb{R}_{+}^{k}$. If $(\bar{x}, \bar{u})$ is an optimal process of $(P(w))$, then it is a weak Pareto optimal process of $(M C P)$.

Proof Similar to the proof of Theorem 10.

Theorem 12 Let $0 \neq w \in \mathbb{R}_{+}^{k}$ and $(\bar{x}, \bar{u})$ be an extremal process of $(P(w))$. Assume that $(H(w))$ is satisfied and $(P(w))$ is MP-pseudoinvex at $(\bar{x}, \bar{u})$, according to Definition 2 . Then $(\bar{x}, \bar{u})$ is a Pareto optimal process of $(M C P)$.

Proof Since $(\mathrm{P}(w))$ is MP-pseudoinvex, $(\bar{x}, \bar{u})$ is an optimal process. Now the result follows from the Theorem 10.

Theorem 13 Let $(\bar{x}, \bar{u})$ be an extremal process of $(M C P)$ with multipliers $(\lambda, \omega, p) \in$ $\Lambda(\bar{x}, \bar{u})$. If $(P(\omega))$ is MP-pseudoinvex according to Definition 2 , then $(\bar{x}, \bar{u})$ is an optimal process of $(P(\omega))$.

Proof By the maximum principle, every extremal process of (MCP) is an extremal process of $(\mathrm{P}(\omega))$, where $\omega$ is the associated multiplier. Hence $(\bar{x}, \bar{u})$ is an extremal process of $(\mathrm{P}(\omega))$ and, since $(\mathrm{P}(\omega))$ is MP-pseudoinvex, by Theorem 7, $(\bar{x}, \bar{u})$ is an optimal process of $(\mathrm{P}(\omega))$.

In the last two theorems, in the case when $(\bar{x}, \bar{u})$ is a normal extremal process of $(\mathrm{P}(w))$ and (MCP), respectively, we can replace the hypothesis of MP-pseudoinvexity at $(\bar{x}, \bar{u})$ according to Definition 2 by

(i) normal MP-pseudoinvexity at $(\bar{x}, \bar{u})$ according to Definition 2, or

(ii) MP-pseudoinvexity at $(\bar{x}, \bar{u})$ according to Definition 1 .

If we use (ii) as hypothesis, then we should utilize Theorem 3 in the proofs.

Let $(\bar{x}, \bar{u})$ be a weak Pareto optimal process of (MCP). Then it is an extremal process of (MCP) with multipliers, say $(\lambda, \omega, p) \in \Lambda(\bar{x}, \bar{u})$. Therefore, $(\bar{x}, \bar{u})$ is also an extremal process of $(\mathrm{P}(\omega))$. If $(\mathrm{P}(\omega))$ is MP-pseudoinvex at $(\bar{x}, \bar{u})$, according to Definition 2, then we can say that there exists a nonzero vector $\omega \in \mathbb{R}_{+}^{k}$ such that $(\bar{x}, \bar{u})$ is an optimal process of $(\mathrm{P}(\omega))$.

Here, if $(\bar{x}, \bar{u})$ turns to be a normal extremal process of (MCP), then the MP-pseudoinvexity of $(\mathrm{P}(\omega))$ at $(\bar{x}, \bar{u})$ can be replaced by hypothesis (i) or (ii) from the remark above.

In the sequel we define the concept of MP-invexity for (MCP).

Definition 3 Let $(\bar{x}, \bar{u})$ be a feasible process. (MCP) is said to be MP-invex at $(\bar{x}, \bar{u})$ if given $\alpha \in(0,1), \gamma=\left[\begin{array}{llll}\gamma_{1} & \gamma_{2} & \ldots & \gamma_{k}\end{array}\right]^{\top} \in D g(\bar{x}(S), \bar{x}(T))$ and $\zeta(t) \in D_{x} f(t, \bar{x}(t), \bar{u}(t))$ a.e. $t \in[S, T]$, there exist maps $(\eta, \xi) \in \mathcal{F}_{\alpha}(\bar{x}, \bar{u} ; \zeta)$ such that, for each $j \in\{1,2, \ldots, k\}$,

$$
g_{j}(x(S), x(T))-g_{j}(\bar{x}(S), \bar{x}(T)) \geq \gamma_{j} \cdot(\eta(S), \eta(T))
$$

for all feasible processes $(x, u)$.

(MCP) is said to be MP-invex if it is MP-invex at every feasible process $(\bar{x}, \bar{u})$.

Theorem 14 Let $(\bar{x}, \bar{u})$ be a normal extremal process of $(M C P)$. Assume that $(M C P)$ is $M P$ invex at $(\bar{x}, \bar{u})$ according to Definition 3. Then there exists a nonzero vector $w \in \mathbb{R}_{+}^{k}$ such that $(\bar{x}, \bar{u})$ is an optimal process of $(P(w))$. 
Proof Provided $(\bar{x}, \bar{u})$ is a normal extremal process of (MCP), there exist multipliers $(\lambda, \omega, p) \in \Lambda(\bar{x}, \bar{u})$, with $\lambda=1$, which satisfy,

$$
\begin{aligned}
& -\dot{p}(t)=\zeta(t)^{\top} p(t) \quad \text { a.e. } t \in[S, T], \\
& (p(S),-p(T))=\omega^{\top} \gamma+\left(v_{0}, v_{1}\right), \\
& \max _{u \in U(t)} \mathcal{H}(t, \bar{x}(t), p(t), u)=\mathcal{H}(t, \bar{x}(t), p(t), \bar{u}(t)) \quad \text { a.e. } t \in[S, T], \\
& \|\omega\|=1, \quad \omega \geq 0,
\end{aligned}
$$

for some $\zeta(t) \in D_{x} f(t, \bar{x}(t), \bar{u}(t))$ a.e. $t \in[S, T], \gamma=\left[\gamma_{1} \gamma_{2} \ldots \gamma_{k}\right]^{\top} \in D g(\bar{x}(S), \bar{x}(T))$ and $\left(v_{0}, v_{1}\right) \in N_{C}(\bar{x}(S), \bar{x}(T))$.

By the MP-invexity of (MCP) there exist maps $\eta$ and $\xi$ obeying (24) along with

$$
\begin{aligned}
& \dot{\eta}(t)=\zeta(t) \eta(t)+\Delta_{\alpha} f(t, \bar{x}(t), \bar{u}(t))(\xi(t)) \quad \text { a.e. } t \in[S, T], \\
& (\eta(S), \eta(T)) \in \bar{T}_{C}(\bar{x}(S), \bar{x}(T)), \\
& \bar{u}(t)+\alpha \xi(t) \in U(t) \quad \text { a.e. } t \in[S, T] .
\end{aligned}
$$

Suppose that $(\bar{x}, \bar{u})$ is not an optimal process of $(\mathrm{P}(w))$ for any $w \in \mathbb{R}_{+}^{k}$. Then, particularly, it is not an optimal process of $(\mathrm{P}(\omega))$, so that there exists a feasible process $(x, u)$ such that $g_{0}(x(S), x(T))=\omega \cdot g(x(S), x(T))<g_{0}(\bar{x}(S), \bar{x}(T))=\omega \cdot g(\bar{x}(S), \bar{x}(T))$. By (24) one has

$$
\left[\omega^{\top} \gamma\right] \cdot(\eta(S), \eta(T))<0,
$$

so that, by (25),

$$
\left[\omega^{\top} \gamma\right] \cdot(\eta(S), \eta(T))+\int_{S}^{T} p(t) \cdot\left[\dot{\eta}(t)-\zeta(t) \eta(t)-\Delta_{\alpha} f(t, \bar{x}(t), \bar{u}(t))(\xi(t))\right] \mathrm{d} t<0 .
$$

Now proceeding as in the proof of Theorem 2 a contradiction is gotten.

Corollary 8 Let $(\bar{x}, \bar{u})$ be a weak Pareto optimal process of $(M C P)$. Assume that $(M C P)$ is normal and MP-invex at $(\bar{x}, \bar{u})$ according to Definition 3. Then there exists a nonzero vector $w \in \mathbb{R}_{+}^{k}$ such that $(\bar{x}, \bar{u})$ is an optimal process of $(P(w))$.

Corollary 8 generalizes for the nonsmooth case the corresponding result in [22], where smooth problems were tackled.

Definition 4 Let $(\bar{x}, \bar{u})$ be a feasible process. (MCP) is said to be $M P$-invex at $(\bar{x}, \bar{u})$ if given $\alpha \in(0,1)$ and $(\lambda, \omega, p) \in \Lambda(\bar{x}, \bar{u})$, there exist maps $\eta:[S, T] \rightarrow \mathbb{R}^{n}$ and $\xi:[S, T] \rightarrow \mathbb{R}^{m}$ such that

$$
\omega \cdot g(x(S), x(T))-\omega \cdot g(\bar{x}(S), \bar{x}(T)) \geq \mathcal{J}_{\alpha}(\bar{x}, \bar{u} ; \lambda, \omega, p)(\eta, \xi)
$$

for all feasible processes $(x, u)$, where

$$
\begin{aligned}
& (\eta(S), \eta(T)) \in \bar{T}_{C}(\bar{x}(S), \bar{x}(T)), \\
& \bar{u}(t)+\alpha \xi(t) \in U(t) \quad \text { a.e. } t \in[S, T] .
\end{aligned}
$$

When the conditions above hold true only with $\lambda=1$, (MCP) is said to be normal MP-invex at $(\bar{x}, \bar{u})$.

(MCP) is said to be (normal) MP-invex if it is (normal) MP-invex at every feasible process.

Theorem 15 Let $(\bar{x}, \bar{u})$ be a (normal) extremal process of $(M C P)$. Assume that $(M C P)$ is (normal) MP-invex at $(\bar{x}, \bar{u})$ according to Definition 4 . Then there exists a nonzero vector $w \in \mathbb{R}_{+}^{k}$ such that $(\bar{x}, \bar{u})$ is an optimal process of $(P(w))$. 
Proof Since $(\bar{x}, \bar{u})$ is an extremal process of (MCP), there exist multipliers, say $(\lambda, \omega, p) \in$ $\Lambda(\bar{x}, \bar{u})$, which satisfy

$$
\max _{u \in U(t)} \mathcal{H}(t, \bar{x}(t), p(t), u)=\mathcal{H}(t, \bar{x}(t), p(t), \bar{u}(t)) \quad \text { a.e. } t \in[S, T] .
$$

From the MP-invexity of (MCP) we know that there exist maps $\eta$ and $\xi$ obeying (28)-(30).

Suppose that there does not exist a nonzero vector $w \in \mathbb{R}_{+}^{k}$ for which $(\bar{x}, \bar{u})$ is an optimal process of $(\mathrm{P}(w))$. So, particularly, it is not an optimal process of $(\mathrm{P}(\omega))$. Hence, there exists a feasible process $(x, u)$ such that $g_{0}(x(S), x(T))=\omega \cdot g(x(S), x(T))<g_{0}(\bar{x}(S), \bar{x}(T))=$ $\omega \cdot g(\bar{x}(S), \bar{x}(T))$. From (28) one sees that

$$
\lambda\left[\omega^{\top} \gamma\right] \cdot(\eta(S), \eta(T))+\int_{S}^{T} p(t) \cdot\left[\dot{\eta}(t)-\zeta(t) \eta(t)-\Delta_{\alpha} f(t, \bar{x}(t), \bar{u}(t))(\xi(t))\right] \mathrm{d} t<0 .
$$

Proceeding as in the proof of Theorem 2 we come to a contradiction.

Corollary 9 Let $(\bar{x}, \bar{u})$ be a (normal) weak Pareto optimal process of $(M C P)$. Assume that $(M C P)$ is (normal) MP-invex at $(\bar{x}, \bar{u})$ according to Definition 4 . Then there exists a nonzero vector $w \in \mathbb{R}_{+}^{k}$ such that $(\bar{x}, \bar{u})$ is an optimal process of $(P(w))$.

Corollary 10 Let $(\bar{x}, \bar{u})$ be a feasible process. Assume that $(M C P)$ is MP-invex at $(\bar{x}, \bar{u})$ according to Definition 3 or 4 . Then $(\bar{x}, \bar{u})$ is a weak Pareto optimal process of $(M C P)$ if, and only if, it is an optimal process of $(P(w))$ for some $0 \neq w \in \mathbb{R}_{+}^{k}$.

As every Pareto optimal process is a weak Pareto optimal process of (MCP), Corollaries 8 and 9 are valid assuming that $(\bar{x}, \bar{u})$ is a Pareto optimal process. However, Corollary 10 is not true unless it is assumed that $(\mathrm{H}(w))$ is satisfied at every $0 \neq w \in \mathbb{R}_{+}^{k}$.

\section{Conclusion}

An extremal control process is a process that satisfies the maximum principle of Pontryagin. Nevertheless, an extremal process may not be optimal. It is the case for control problems whose dynamics are linear and the costs are convex functionals. This sufficiency of the maximum principle for linear quadratic problems lead Pontryagin to think, at first, that the maximum principle conditions were sufficient for nonlinear problems. However, as expected, in general, the maximum principle is only a set of necessary conditions for optimality. Since then, a question that remained open until recently was: what are the weakest assumptions and conditions that should be imposed to the control problem data in order that the maximum principle becomes automatically a set of sufficient conditions of optimality? A complete answer to that question has been addressed in this paper for fixed time control problems with multiple objectives. It was shown that the class of MP-pseudoinvex optimal control problems is the largest class of nonsmooth control problems in which every process that satisfies the maximum principle is optimal. It was also shown that the class of MP-invex optimal control problems is the most general class of multiobjective problems in which resolving a vectorial problem is equivalent to solving a related scalar one.

As future research on these issues, we will work on generalizing our results to free time problems with state constraints.

Acknowledgments The authors were supported by Grant 2013/07375-0, São Paulo Research Foundation (FAPESP) and by Grants 457785/2014-4, 479109/2013-3, and 309335/2012-4, National Council for Scientific and Technological Development (CNPq). 


\section{References}

1. Antczak, T.: On G-invex multiobjective programming. Part I. Optimality. J. Glob. Optim. 43, 97-109 (2009)

2. Antczak, T.: Proper efficiency conditions and duality results for nonsmooth vector optimization in Banach spaces under $(\Phi, \rho)$-invexity. Nonlin. Anal. 75(6), 3107-3121 (2012)

3. Arana-Jiménez, M., Osuna-Gómez, R., Ruiz-Garzón, G., Rojas-Medar, M.: On variational problems: characterization of solutions and duality. J. Math. Anal. Appl. 311, 1-12 (2005)

4. Arana-Jiménez, M., Osuna-Gómez, R., Rufián-Lizana, A., Ruiz-Garzón, G.: KT-invex control problem. Appl. Math. Comput. 197, 489-496 (2008)

5. Arana-Jiménez, M., Hernández-Jiménez, B., Ruiz-Garzón, G., Rufián-Lizana, A.: FJ-invex control problem. Appl. Math. Lett. 22, 1887-1891 (2009)

6. Arana-Jiménez, M., Rufián-Lizana, A., Ruiz-Garzón, G., Osuna-Gómez, R.: Efficient solutions in V-KTpseudoinvex multiobjective control problems: a characterization. Appl. Math. Comput. 215, 441-448 (2009)

7. Arana-Jiménez, M., Ruiz-Garzón, G., Osuna-Gómez, R., Rufián-Lizana, A.: Weakly efficient solutions and pseudoinvexity in multi objective control problems. Nonlin. Anal. 73, 1792-1801 (2010)

8. Arana-Jiménez, M., Ruiz-Garzón, G., Rufián-Lizana, A., Osuna-Gómez, R.: Weak efficiency in multiobjective variational problems under generalized convexity. J. Glob. Optim. 52, 109-121 (2012)

9. Arana-Jiménez, M., Ruiz-Garzón, G., Osuna-Gómez, R., Hernández-Jiménez, B.: Duality and a characterization of pseudoinvexity for Pareto and weak Pareto solutions in nondifferentiable multiobjective programming. J. Optim. Theory Appl. 156, 266-277 (2013)

10. Arutyunov, A.V., Karamzin, D.Y., Pereŭra, F.: R. V. Gamkrelidze's maximum principle for optimal control problems with bounded phase coordinates and its relation to other optimality conditions. Dokl. Akad. Nauk 436(6), 738-742 (2011)

11. Arutyunov, A.V., Karamzin, D.Y., Pereira, F.L.: The maximum principle for optimal control problems with state constraints by R. V. Gamkrelidze: revisited. J. Optim. Theory Appl. 149(3), 474-493 (2011)

12. Bellaassali, S., Jourani, A.: Necessary optimality conditions in multiobjective dynamic optimization. SIAM J. Control Optim. 42, 2043-2061 (2004)

13. Bonnel, H., Kaya, C.Y.: Optimization over the efficient set of multi-objective convex optimal control problems. J. Optim. Theory Appl. 147(1), 93-112 (2010)

14. Burai, P.: Necessary and sufficient condition on global optimality without convexity and second order differentiability. Optim. Lett. 7(5), 903-911 (2013)

15. Burai, P.: Local-global minimum property in unconstrained minimization problems. J. Optim. Theory Appl. 162(1), 34-46 (2014)

16. Clarke, F.H.: Optimization and Nonsmooth Analysis, Classics in Applied Mathematics, vol. 5. SIAM, Philadelphia (1990)

17. Clarke, F.H., Ledyaev, Y.S., Stern, R.J., Wolenski, P.R.: Nonsmooth Analysis and Control Theory, Graduate Texts in Mathematics, vol. 178. Springer, New York (1998)

18. Clarke, F.: Functional Analysis, Calculus of Variations and Optimal Control, Graduate Texts in Mathematics, vol. 264. Springer, London (2013)

19. Craven, B.D., Glover, B.M.: Invex functions and duality. J. Aust. Math. Soc. Ser. A 39, 1-20 (1985)

20. de Oliveira, V.A., Rojas-Medar, M.A.: Continuous-time multiobjective optimization problems via invexity. Abstr. Appl. Anal. 2007, Art. ID 61296, 11 (2007)

21. de Oliveira, V.A., Rojas-Medar, M.A., Brandão, A.J.V.: A note on KKT-invexity in nonsmooth continuoustime optimization. Proyecciones 26, 269-279 (2007)

22. de Oliveira, V.A., Silva, G.N., Rojas-Medar, M.A.: A class of multiobjective control problems. Optim. Control Appl. Methods 30, 77-86 (2009)

23. de Oliveira, V.A., Silva, G.N., Rojas-Medar, M.A.: KT-invexity in optimal control problems. Nonlin. Anal. Theory Methods Appl. 71, 4790-4797 (2009)

24. de Oliveira, V.A., dos Santos, L.B., Osuna-Gómez, R., Rojas-Medar, M.A.: Optimality conditions for nonlinear infinite programming problems. Optim. Lett. 9, 1131-1147 (2015). doi:10.1007/ s11590-014-0808-9

25. de Oliveira, V.A., Rojas-Medar, M.A.: Continuous-time optimization problems involving invex functions. J. Math. Anal. Appl. 327, 1320-1334 (2007)

26. de Oliveira, V.A., Rojas-Medar, M.A.: Multi-objective infinite programming. Comput. Math. Appl. 55, 1907-1922 (2008)

27. de Oliveira, V.A., Silva, G.N.: New optimality conditions for nonsmooth control problems. J. Glob. Optim. 57, 1465-1484 (2013) 
28. Dinuzzo, F., Ong, C., Gehler, P., Pillonetto, G.: Learning output kernels with block coordinate descent. In: Proceedings of the 28th International Conference on Machine Learning, ICML 2011, pp. 49-56 (2011)

29. Gamkrelidze, R.V.: Principles of optimal control theory (transl. from the Russian by K. Malowski. Transl. ed. by and with a foreword by L. D. Berkovitz). In Mathematical Concepts and Methods in Science and Engineering, vol. 7. Plenum Press, New York, London (1978)

30. Hanson, M.A.: On sufficiency of the Kuhn-Tucker conditions. J. Math. Anal. Appl. 80, 545-550 (1981)

31. Hanson, M.A.: Invexity and the Kuhn-Tucker theorem. J. Math. Anal. Appl. 236, 594-604 (1999)

32. Hernández-Jiménez, B., Rojas-Medar, M.A., Osuna-Gómez, R., Beato-Moreno, A.: Generalized convexity in non-regular programming problems with inequality-type constraints. J. Math. Anal. Appl. 352, 604-613 (2009)

33. Hernández-Jiménez, B., Osuna-Gómez, R., Arana-Jiménez, M., Ruiz-Garzón, G.: Generalized convexity and efficiency for non-regular multiobjective programming problems with inequality-type constraints. Nonlin. Anal. Theory Methods Appl. 73, 2463-2475 (2010)

34. Hernández-Jiménez, B., Rojas-Medar, M.A., Osuna-Gómez, R., Rufián-Lizana, A.: Characterization of weakly efficient solutions for non-regular multiobjective programming problems with inequality-type constraints. J. Convex Anal. 18, 749-768 (2011)

35. Hernández-Jiménez, B., Osuna-Gómez, R., Rojas-Medar, M.A., dos Santos, L.B.: Generalized convexity for non-regular optimization problems with conic constraints. J. Glob. Optim. 57, 649-662 (2013)

36. Karamzin, D.Y., de Oliveira, V.A., Pereira, F.L., Silva, G.N.: On some extension of optimal control theory. Eur. J. Control 20(6), 284-291 (2014)

37. Kenan, Z., Lok, T.: Optimal power allocation for relayed transmission through a mobile relay node. In: IEEE Vehicular Technology Conference. Article number 5494018 (2010)

38. Kenan, Z., Lok, T.: Power control for uplink transmission with mobile users. IEEE Trans. Veh. Technol. 60(5), 2117-2127 (2011)

39. Kien, B.T., Wong, N.C., Yao, J.C.: Necessary conditions for multiobjective optimal control problems with free end-time. SIAM J. Control Optim. 47(5), 2251-2274 (2008)

40. Luc, D.T.: Generalized convexity in vector optimization. In: Hadjisavvas, N., Komlsi, S., Schaible, S. (eds.) Handbook of Generalized Convexity and Generalized Monotonicity, Nonconvex Optimization and Its Applications, vol. 76, pp. 195-236. Springer, New York (2005)

41. Martin, D.H.: The essence of invexity. J. Optim. Theory Appl. 47, 65-76 (1985)

42. Mishra, S.K., Wang, S., Lai, K.K.: V-Invex Functions and Vector Optimization, Springer Optimization and Its Applications, vol. 14. Springer, New York (2008)

43. Mishra, S.K., Wang, S., Lai, K.K.: Generalized Convexity and Vector Optimization, Nonconvex Optimization and Its Applications, vol. 90. Springer, Berlin (2009)

44. Mordukhovich, B.S.: Variational Analysis and Generalized Differentiation I. Basic Theory, Grundlehren der Mathematischen Wissenschaften [Fundamental Principles of Mathematical Sciences], vol. 330. Springer, Berlin (2006)

45. Mordukhovich, B.S.: Variational Analysis and Generalized Differentiation II. Applications, Grundlehren der Mathematischen Wissenschaften [Fundamental Principles of Mathematical Sciences], vol. 331. Springer, Berlin (2006)

46. Nickisch, H., Seeger, M.: Multiple kernel learning: A unifying probabilistic viewpoint (2011). arXiv: 1103.0897

47. Osuna-Gómez, R., Rufián-Lizana, A., Ruíz-Canales, P.: Invex functions and generalized convexity in multiobjective programming. J. Optim. Theory Appl. 98, 651-661 (1998)

48. Osuna-Gómez, R., Beato-Moreno, A., Rufián-Lizana, A.: Generalized convexity in multiobjective programming. J. Math. Anal. Appl. 233, 205-220 (1999)

49. Pontryagin, L.S., Boltyanskii, V.G., Gamkrelidze, R.V., Mishchenko, E.F.: The mathematical theory of optimal processes (translated by D. E. Brown. A Pergamon Press Book). The Macmillan Co., New York (1964)

50. Pontryagin, L., Boltyanskij, V., Gamkrelidze, R., Mishchenko, E.: Selected works, vol. 4. The mathematical theory of optimal processes. Ed. and with a preface by R. V. Gamkrelidze (transl. from the Russian by K. N. Trirogoff. Transl. ed. by L. W. Neustadt. With a preface by L. W. Neustadt and K. N. Trirogoff. Reprint of the 1962 Engl. translation. Classics of Soviet Mathematics). Gordon and Breach Science Publishers, New York, p. xxiv (1986)

51. Reiland, T.W.: Nonsmooth invexity. Bull. Aust. Math. Soc. 42, 437-446 (1990)

52. Rockafellar, R.T., Wets, R.J.B.: Variational Analysis, Grundlehren der Mathematischen Wissenschaften [Fundamental Principles of Mathematical Sciences], vol. 317. Springer, Berlin (1998)

53. Sach, P.H., Kim, D.S., Lee, G.M.: Generalized convexity and nonsmooth problems of vector optimization. J. Glob. Optim. 31, 383-403 (2005) 
54. Silva, G.N., Vinter, R.B.: Necessary conditions for optimal impulsive control problems. SIAM J. Control Optim. 35(6), 1829-1846 (1997)

55. Slimani, H., Radjef, M.S.: Multiobjective Programming Under Generalized Invexity. Lap Lambert Academic Publishing, Saarbrücken (2010)

56. Slimani, H., Radjef, M.S.: Nondifferentiable multiobjective programming under generalized $d_{I}$-invexity. Eur. J. Oper. Res. 202(1), 32-41 (2010)

57. Syed, M., Pardalos, P., Principe, J.: Invexity of the minimum error entropy criterion. IEEE Signal Process. Lett. 20(12), 1159-1162 (2013)

58. Vinter, R.B.: Optim. Control. Birkhäuser, Boston (2000)

59. Zhao, F.A.: On sufficiency of the Kuhn-Tucker conditions in nondifferentiable programming. Bull. Aust. Math. Soc. 46, 385-389 (1992)

60. Zhu, Q.J.: Hamiltonian necessary conditions for a multiobjective optimal control problem with endpoint constraints. SIAM J. Control Optim. 39(1), 97-112 (2000) 